\title{
PREDICTING MONTHLY INPATIENT VOLUMES IN HOSPITALS
}

\author{
Samira Rasouli ${ }^{1}$, Hamed Tabesh ${ }^{2}$, Kobra Etminani $^{3, *}$
}

1: MSc Student, Medical Informatics, Student Research Committee, Medical Informatics Department, Faculty of Medicine, Mashhad University of Medical Sciences, Mashhad, Iran

2: Assistant Professor, Biostatistics, Medical Informatics Department, Faculty of Medicine, Mashhad University of Medical Sciences, Mashhad, Iran

3: Assistant Professor, Medical Informatics, Medical Informatics Department, Faculty of Medicine, Mashhad University of Medical Sciences, Mashhad, Iran

Correspondence:

Tel: +98.09155118312, E-mail: Etminanik@mums.ac.ir

\section{TYPE OF ARTICLE: CONFERENCE ABSTRACT}

\begin{abstract}
Introduction: The study and analysis of hospital processes have become a necessity for overall performance improvement of the hospital systems through developing improved policies and decisions. In this context, management and analysis of patient volume is an important factor. The goal of this paper is to evaluate the use of multiple exponential smoothing forecasting methods to predict monthly hospital inpatient volume at 10 different hospitals and to compare the accuracy performance of these methods.

Methods: Monthly inpatient admissions at 10 different hospitals were collected for the period of March 2004 through March 2016. We evaluated the use of simple exponential smoothing (SES), Holt-Winters seasonal method, Holt and exponential smoothing state space models to forecast monthly inpatient volume at each hospital. Forecasts were made for horizons ranging from one to three months ahead. The performance of the models was measured based on the mean absolute percentage error (MAPE) accuracy measure.

Results: In this study, the Holt-Winters seasonal method provided optimal forecasting performance for eight hospitals. Also, Holt and Exponential smoothing state space methods had the best results for two hospitals. In total, best forecasting models of all 10 hospitals had a MAPE ranging from $1.06 \%$ to $5.95 \%$, indicating an accurate prediction.

Conclusion: The Holt-Winters seasonal method provided useful predictions of the number of monthly inpatient volume. Applied methods in this study may assist other hospitals and emergency departments for forecasting purposes.
\end{abstract}

KEYWORDS: Time series forecasting, Exponential smoothing, Hospital, Inpatient volume

\section{Abstracts of First National Congress of Medical Informatics, Mashhad, Iran, February 2017}

(C) 2017 The Authors. This is an open access article under the terms of the Creative Commons Attribution-NonCommercialNoDerivs License, which permits use and distribution in any medium, provided the original work is properly cited, the use is non-commercial and no modifications or adaptations are made. 